\title{
Bimodal Cochlear Implant Listeners' Ability to Perceive Minimal Audible Angle Differences
}

DOI: $10.3766 /$ jaaa. 17012

\author{
Ashley Zaleski-King* \\ Matthew J. Goupell† \\ Dragana Barac-Cikoja* \\ Matthew Bakke*
}

\begin{abstract}
Background: Bilateral inputs should ideally improve sound localization and speech understanding in noise. However, for many bimodal listeners [i.e., individuals using a cochlear implant $(\mathrm{Cl})$ with a contralateral hearing aid $(\mathrm{HA})]$, such bilateral benefits are at best, inconsistent. The degree to which clinically available $\mathrm{HA}$ and $\mathrm{Cl}$ devices can function together to preserve interaural time and level differences (ITDs and ILDs, respectively) enough to support the localization of sound sources is a question with important ramifications for speech understanding in complex acoustic environments.
\end{abstract}

Purpose: To determine if bimodal listeners are sensitive to changes in spatial location in a minimum audible angle (MAA) task.

Research Design: Repeated-measures design.

Study Sample: Seven adult bimodal Cl users (28-62 years). All listeners reported regular use of digital HA technology in the nonimplanted ear.

Data Collection and Analysis: Seven bimodal listeners were asked to balance the loudness of prerecorded single syllable utterances. The loudness-balanced stimuli were then presented via direct audio inputs of the two devices with an ITD applied. The task of the listener was to determine the perceived difference in processing delay (the interdevice delay [IDD]) between the $\mathrm{Cl}$ and $\mathrm{HA}$ devices. Finally, virtual free-field MAA performance was measured for different spatial locations both with and without inclusion of the IDD correction, which was added with the intent to perceptually synchronize the devices.

Results: During the loudness-balancing task, all listeners required increased acoustic input to the HA relative to the $\mathrm{Cl}$ most comfortable level to achieve equal interaural loudness. During the ITD task, three listeners could perceive changes in intracranial position by distinguishing sounds coming from the left or from the right hemifield; when the $\mathrm{Cl}$ was delayed by $0.73,0.67$, or $1.7 \mathrm{msec}$, the signal lateralized from one side to the other. When MAA localization performance was assessed, only three of the seven listeners consistently achieved above-chance performance, even when an IDD correction was included. It is not clear whether the listeners who were able to consistently complete the MAA task did so via binaural comparison or by extracting monaural loudness cues. Four listeners could not perform the MAA task, even though they could have used a monaural loudness cue strategy.

Conclusions: These data suggest that sound localization is extremely difficult for most bimodal listeners. This difficulty does not seem to be caused by large loudness imbalances and IDDs. Sound localization is best when performed via a binaural comparison, where frequency-matched inputs convey ITD and ILD information. Although low-frequency acoustic amplification with a HA when combined with a $\mathrm{Cl}$ may produce an overlapping region of frequency-matched inputs and thus provide an opportunity for binaural comparisons for some bimodal listeners, our study showed that this may not be beneficial or useful for spatial location discrimination tasks. The inability of our listeners to use monaural-level cues to

*Department of Speech and Hearing Sciences, Gallaudet University, Washington, DC; †Department of Hearing and Speech Sciences, University of Maryland College Park, College Park, MD

Corresponding author: Ashley Zaleski-King, Department of Speech and Hearing Sciences, Gallaudet University, Washington, DC 20002; Email: Ashley.zaleski@gmail.com

This study was partially supported by NIH-NIDCD Grant R01 DC014948 (M.J.G.). 
perform the MAA task highlights the difficulty of using a $\mathrm{HA}$ and $\mathrm{Cl}$ together to glean information on the direction of a sound source.

Key Words: cochlear implants, hearing aids and assistive listening devices, hearing science

Abbreviations: $\mathrm{Cl}=$ cochlear implant; $\mathrm{HA}$ = hearing aid; IDD = inter-device delay; ILD = interaural level difference; ITD = interaural time differences; MAA = minimum audible angle; $\mathrm{MCL}=$ most comfortable level; PTA = pure tone average; SD = standard deviation

\section{INTRODUCTION}

$\mathrm{E}$ xpanded cochlear-implant (CI) candidacy criteria have resulted in an increasing number of unilateral CI users with usable residual hearing in the nonimplanted ear. For many listeners with significant hearing loss, this residual hearing may be accessed with a hearing aid (HA; Most et al, 2011) or, in rarer cases, for listeners with minimal hearing loss in one ear, without a HA (Sampaio et al, 2011). This configuration, consisting of a CI and a contralateral HA, has been described as bimodal listening. It has been estimated that approximately $60 \%$ of all adult CI recipients today have aidable low-frequency contralateral hearing thresholds (80- to 85-dB HL at $250 \mathrm{~Hz}$; Dorman and Gifford, 2010). For such individuals, it has been recommended that the CI be supplemented with contralateral acoustic input for improved speech understanding and spatial localization (Ching et al, 2004; Ching et al, 2007; Most et al, 2011). Some bimodal listeners can take advantage of spatial cues when speech sources are spatially separated through (a) the head shadow effect, a monaural phenomenon by which the spatial separation of sources results in an ear with a more favorable signalto-noise ratio and, potentially, (b) binaural squelch, an effect arising from computation of interaural differences which allows the listener to selectively attend to a speech signal (Dunn et al, 2005; Morera et al, 2005; van Hoesel, 2012). When speech and noise are colocated in space, some bimodal listeners are also able to benefit from binaural summation, increased perceptual loudness resulting from central integration of speech signals (Shallop et al, 1992; Ching et al, 2004; Olson and Shinn, 2008; Schafer et al, 2011; Kokkinakis and Pak, 2014). Many of these benefits have been demonstrated in optimized, directly controlled laboratory conditions (Francart et al, 2009b; Francart et al, 2011a,b) and in comparison to monaural listening with either a CI or HA alone in the contralateral ear.

The purpose of this study was to investigate the degree to which bimodal listeners can identify differences in broadband sound source location using clinical CI and HA devices. Based on the known importance of interaural cues, we aimed to determine if this localization ability might be improved after accounting for differences in HA and CI timing delays.

\section{Potential Bimodal Listening Benefit}

The use of a HA in the nonimplanted ear allows for acoustic stimulation primarily to low frequencies, which may improve the quality of sounds, provide useful pitch information, and convey prosodic information that can support the perceptual organization of sound sources (Armstrong et al, 1997; Hamzavi et al, 2004; Gfeller et al, 2007; Nittrouer and Chapman, 2009; Sucher and McDermott, 2009; Most et al, 2011; Zhang et al, 2013; Crew et al, 2015; Bernstein et al, 2016; Devocht et al, 2017). By contrast, CI input provides frequency information between about 100 and $8000 \mathrm{~Hz}$, although this information is typically delivered to neurons tuned to middle and high-frequency regions of the cochlea (Landsberger et al, 2015). One potential benefit of interaural overlap is improved sound localization through binaural processing.

Ching et al (2007) reviewed four studies that investigated sound localization performance advantages for children and adults using bimodal hearing devices. Across the studies that included adults listening to broadband noise or speech stimuli, about half of the listeners showed a significant improvement in sound localization when comparing unilateral and bilateral conditions; the other half showed equivalent performance. No relationship was identified between listeners' residual hearing pure-tone average (PTA) in the HA ear and the degree of benefit in these studies; however, in most of the studies included, the bimodal listeners' acoustic ear hearing was limited (averaged PTA $\geq 90 \mathrm{~dB} \mathrm{HL}$ ). In another bimodal listener study including 11 listeners, localization performance ranged from high accuracy (e.g., $4.5^{\circ}$ root-mean square error), accuracy limited to left-right side discrimination, or no localization ability at all (Seeber et al, 2004). In contrast to Ching (2007), Seeber et al found that the three listeners who benefitted most from the bimodal configuration also had the highest amount of residual hearing. These differences suggest a need for a more specific understanding of the optimum bimodal listening mode and strategy.

To localize a sound source in the horizontal plane, individuals with normal hearing typically use differences in the timing and intensity characteristics of sound in one ear relative to the other ear. Interaural time differences (ITDs) are most salient for sounds with 
low-frequency content $(<1500 \mathrm{~Hz}$; Wightman and Kistler, 1992; Macpherson and Middlebrooks, 2002). Interaural level differences (ILDs) are derived from the head casting an "acoustic shadow" that results in differences in intensity between the ears. ILDs increase with increasing frequency (Feddersen et al, 1957), but become nonmonotonic at large azimuths between $\sim 1000$ and $4000 \mathrm{~Hz}$ (Macaulay et al, 2010). For a source at $90^{\circ}$, ILDs produced by the head and pinna are approximately $0.7 \mathrm{~dB}$ at $100 \mathrm{~Hz}, 5.2 \mathrm{~dB}$ at $1000 \mathrm{~Hz}$, and $25.1 \mathrm{~dB}$ at $8000 \mathrm{~Hz}$ (Kayser et al, 2009). Although relatively small at low frequencies, this does not preclude listeners from potentially using low-frequency ILDs to localize sounds. Listeners with normal hearing can discriminate approximately 1-dB ILDs over headphones across wide ranges of frequencies (Mills, 1959). When listeners lack interaural symmetry in audible bandwidth (e.g., because of unilateral hearing loss), individuals must rely on monaural spectral shape or loudness information (Shaw and Vaillancourt, 1985). Because many CI and HA processors do not convey salient spectral-shape cues (Majdak et al, 2011), monaural loudness is likely the main cue used for sound localization in such cases.

\section{Bimodal Auditory Cue Preservation}

The degree to which bimodal hearing preserves binaural and/or monaural cues to facilitate sound localization is a question with important ramifications for speech understanding in complex acoustic environments. If some localization cues are preserved, it is not clear whether or not bimodal listeners would use listening strategies that would use them. Usable acoustic hearing above about $1-2 \mathrm{kHz}$ is limited in both ears for most bimodal listeners (Von Ilberg et al, 2011); unaided thresholds tend to be $>90-100 \mathrm{~dB}$ HL (or immeasurable) above this frequency range (Francart and McDermott, 2013). Despite the significant degree of acoustic ear hearing loss, it may be possible that even limited overlapping frequency regions could support binaural computation of ITDs and ILDs, which may improve sound localization performance (Francart et al, 2009b; Veugen et al, 2016b). Sensitivity to ITDs has been demonstrated in bimodal listeners in laboratory conditions using highly controlled stimuli (e.g., pulse trains or noise stimuli with experimental devices under direct computer control of electrode stimulation patterns; Francart and Wouters, 2007; Francart et al, 2009b; 2011b; Francart et al, 2014). However, another study found that even for individuals trained on an ITD task $(\mathrm{n}=3)$, they demonstrated no or little sensitivity to transposed sinusoids or pulse trains (Lenssen et al, 2011). The degree to which available binaural cues may be beneficial for sound source localization may be related to the degree the loudness is balanced between the HA and CI devices (Potts et al, 2009; Veugen et al, 2016a,b). Francart et al (2011a) investigated localization performance of environmental sounds presented in the sound field using bimodal listeners' own devices in comparison to virtual, computer-controlled conditions, with and without ILD enhancement. The mean absolute error averaged over the six listeners ranged from $31^{\circ}, 28.4^{\circ}$, and $20.6^{\circ}$ for sound field, virtual presentation without ILD enhancement, and virtual presentation with ILD enhancement conditions, respectively. The authors suggested that the individual variability in performance may be related to ILD sensitivity, differences in the ability to relate the ILD to spatial location, and the amount of residual hearing. Importantly, this study used level roving to limit the usability of monaural loudness cues to perform the localization task. The effect of ITD changes on localization was not examined because of the lack of synchronization between CI and HA, which likely limits accessibility to ITD from clinical devices.

The degree to which binaural cues may be available might also be influenced by the methods used to fit the CI and HA devices (Potts et al, 2009). In many instances, bimodal listeners are fitted with a standard $\mathrm{HA}$ and a standard CI, without altering either fitting method to accommodate differences in the devices (Francart and McDermott, 2013). One method to perform a bimodal fit with maximal sensitivity to binaural cues would be to reduce loudness differences across ears. Localization performance can improve using devices balanced in loudness relative to unadjusted devices (Ching et al, 2007). Another method to perform a bimodal fitting might be to compensate for differences in HA and CI processing delay times (Francart et al, 2009b). For both CIs and HAs, the processing delay may be subject to both the sampling rate and the algorithm implemented. The delay from a CI varies by device and can range from 5 to $20 \mathrm{msec}$ (Stone and Moore, 1999). HA processing also produces a delay equivalent to the device's delay and the frequency-dependent traveling wave delay (Stone and Moore, 1999). The HA delay is thought to be smaller than the processing delay of the CI processor, resulting in differences of up to tens of milliseconds (Francart et al, 2013). For example, Cochlear-brand CIs include a filtering and coding strategy that causes a delay at the CI ear of about 10.5-12.5 msec relative to simultaneous presentation of the sound to an acoustical ear (van Dijk, Cochlear, private communication, 2015). Delays of $\sim 10 \mathrm{msec}$ are likely not a problem for speech perception (Stone and Moore, 2003), but they are potentially a problem for tasks requiring neural computation of binaural cues.

The purpose of the present study was to investigate functional, real-world benefit of combined HA and CI listening by compensating for interdevice timing and loudness differences. Although previous research has revealed possible localization benefits resulting from loudness balancing (Ching et al, 2007), the effect of 
attempting synchronization on HA and CI device timing has not been systematically addressed. The input level of the HA was first adjusted to match the loudness of the CI input at the most comfortable level (MCL). Once the loudness was balanced between two ears, the perceived difference in processing delay, the interdevice delay (IDD), was assessed using an ITD lateralization task. Minimum audible angle (MAA) localization performance was then measured using a speech stimulus with broadband spectral content to provide an opportunity for acoustic and electric stimulation overlap. It was hypothesized that listeners who could lateralize the signal based on changes in IDD would be better able to discriminate sound source location. Furthermore, it was predicted that this performance might improve when we included an IDD correction to compensate for interaural timing delays.

\section{METHODS AND MATERIALS}

\section{Listeners}

Seven adult bimodal CI users, three males and four females aged 28-62 years, were recruited from the Washington, DC and Baltimore area. Six of these listeners used a Cochlear Americas processor (N5, N = 4; Freedom, $\mathrm{N}=2$ ). One listener used an Advanced Bionics Harmony processor. All listeners reported regular use of digital HA technology in the nonimplanted ear. Reported listening experience and history of each ear are described in Table 1. Unaided, pure-tone audiometric data from the nonimplanted ear in Figure 1 show mild/ moderate to severe $(\mathrm{N}=2)$, moderately severe, flat $(\mathrm{N}=$ 2 ), moderately severe to profound $(\mathrm{N}=1)$, and severe to profound $(\mathrm{N}=2)$ hearing losses. Listeners provided informed consent for participation and were reimbursed for their time. This study was approved by the Gallaudet University Institutional Review Board.

\section{Speech Stimuli}

A speech token was used in this study because a broadband stimulus would ensure excitation of interaurally matched inputs-necessary for binaural cue computation-if such a region exists for each individual bimodal listener. For all tasks, listeners heard a female speaker utter the word "hawed" (Hillenbrand et al, 1995), which is part of a stimulus used in previous research to investigate spectral and temporal sensitivity in CI listeners (Fu et al, 2004) as well as in research investigating auditory training with $\mathrm{CI}$ listeners (Fu et al, 2005). The stimulus was $0.51 \mathrm{sec}$ in duration, had a fundamental frequency $\left(F_{0}\right)$ of $205 \mathrm{~Hz}$, and had formant frequencies of 408, 1042, 1600, and $2803 \mathrm{~Hz}$.

Stimuli were presented via virtual spatialization. This was completed by generating recordings using Knowles Electronic Manikin for Acoustic Research, KEMAR (GRAS Sound \& Vibration A/S) that was placed within a $14 \times 16.25^{\prime}$ room about $1 / 3$ of the way from the nearest the $14^{\prime}$ wall and centered width-wise. The speech stimulus was played through a digital audio compact disc player, amplified to an approximate $25-30 \mathrm{~dB}$ signalto-noise ratio, and routed through a mouth simulator Type 4227 (Brüel \& Kjær, Sound and Vibration Measurement A/S). The mouth simulator was positioned facing the center of an approximate $5^{\prime}$ radius arc in front of the acoustic manikin, which was placed approximately $49^{\prime \prime}$ from the ground. Stimuli were presented at 17 locations spanning the front $180^{\circ}$ in $11.25^{\circ}$ increments along a fixed radius with the acoustic manikin in the center. Recordings were generated using a pair of calibrated B\&K prepolarized condenser microphones (type 4101) placed at the approximate location of a behind-the-ear HA microphone above the acoustic manikin's pinnae (Aronoff et al, 2011). Recordings were amplified and routed to a two-channel compact disc digital audio system, model CDR 308 (Marantz America, Inc.) with 24-bit resolution. Recorded signals were analyzed through Adobe Audition v. 2.0 (Adobe Systems Inc.).

\section{Experimental Setup and Procedure}

Using a Dell Vostro 1220 laptop (Dell Technologies Inc.) with Windows Vista Business OEMAct 32 bit (Microsoft Co.) operating system, applications developed in Psycon software (Kwon, 2012) were used throughout the study. Stereophonic speech stimuli were delivered to the direct audio inputs of the HA and CI processors. The output of

Table 1. Listener Demographic and Hearing History Information (years)

\begin{tabular}{|c|c|c|c|c|c|c|c|c|}
\hline Participant & Sex & Age & Cl Type & Age at Implantation & Duration of $\mathrm{HL}$ & $\mathrm{Cl}$ Experience & HA Experience & Etiology \\
\hline$\overline{\mathrm{B} 1}$ & $M$ & 69 & Harmony & 54 & 64 & 2 & 5 & Unknown \\
\hline B2 & $\mathrm{F}$ & 77 & Freedom & 63 & 52 & 5 & 34 & Unknown \\
\hline B3 & $\mathrm{F}$ & 63 & Nucleus 5 & 60 & 60 & 3 & 60 & Disease \\
\hline B4 & $\mathrm{F}$ & 28 & Freedom & 21 & Congenital & 5 & $27+$ & Genetic \\
\hline B5 & $\mathrm{F}$ & 63 & Nucleus 5 & 47 & Congenital & 16 & $50+$ & Genetic \\
\hline B6 & M & 73 & Nucleus 5 & 72 & 18-20 & 1 & 11 & Unknown \\
\hline B7 & $\mathrm{F}$ & 72 & Nucleus 5 & 70 & 22 & 2 & 22 & Genetic \\
\hline
\end{tabular}



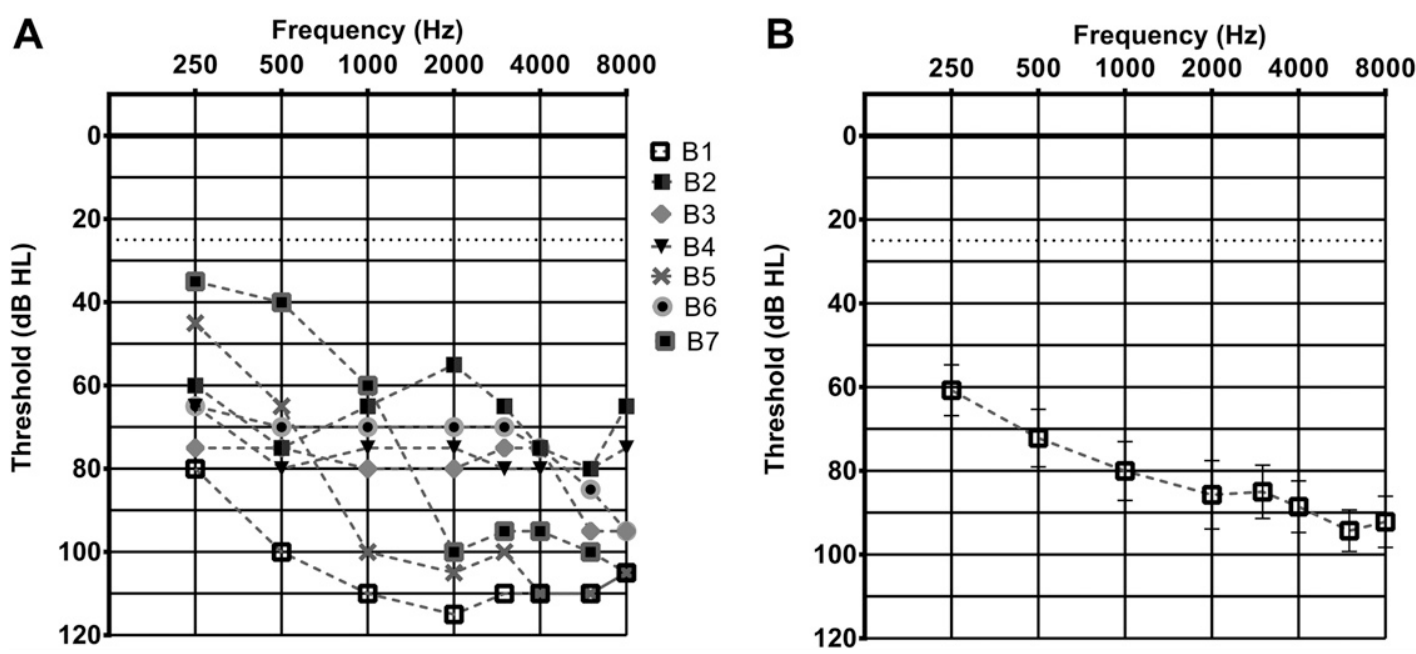

Figure 1. (A) Participant acoustic ear air conduction audiometric thresholds. The dotted line identifies the cutoff for normal hearing sensitivity (25 dB HL). (B) Mean acoustic air conduction threshold with standard error.

each channel was verified using Sennheiser HD201 (Sennheiser Electronic GmbH \& Co. KG) audio headphones. All listeners used a laboratory HA (Phonak Naida UP IX), programmed according to Phonak's recommended fitting algorithm and coupled with a temporary earmold. Nonlinear frequency compression features were disabled. The Naida HA was reprogrammed for each listener based on audiometric data collected (Figure 1) on the initial day of testing. All listeners used their own CI speech processor in the everyday program which was set to receive direct audio input with a 10:1 or an accessory-only mixing ratio.

The experimental procedure consisted of four components, included to (a) determine the MCL with the CI, (b) balance loudness between the HA and CI near the MCL, (c) measure the interaural delay between the HA and the CI, and (d) assess MAA performance. Listeners were not given visual prompts or feedback for any of the four tasks.

\section{MCL Task}

Before completing the MCL task, efforts were made to subjectively validate appropriate HA function and to determine if at everyday volume settings each presentation of the stimulus was audible and comfortable. Then, three tracks of an adaptive procedure were used to estimate each listener's MCL. More than three replications were administered if a listener had difficulty with the task; in these cases, the reported MCL was recorded as an average of MCL estimates from only the last three tracks. A loudness scale ranging from "just noticeable" to "too loud" was used as a visual aid to help listeners understand the task and respond with either "increase" or "decrease" depending on whether the intensity was below or above their MCL. The initial step size of the intensity change in the CI was $5 \mathrm{~dB}$ and was reduced to $2.5 \mathrm{~dB}$ after three reversals. The MCL estimate for each track was determined by an average of at least the last four reversals (or in some cases an even number of reversals greater than four). The reported MCL is an average of MCL values from the last three consecutive tracks.

\section{CI-HA Loudness-Balancing Task}

After the MCL for the CI ear was determined, the acoustic and electric loudness percepts were balanced between HA and CI ears. Using a similar adaptive procedure, the stimuli were presented sequentially to each device, and listeners were asked to identify the ear with the softer presentation. The input level of the HA was increased or decreased (one-up/one-down) based on the listener's response to balance the loudness of the HA level to the CI MCL for all listeners with exception of B6, where the HA was used as the reference and the input level of the CI was adjusted. The initial step size of the intensity change in the HA was $5 \mathrm{~dB}$ and was reduced to $3 \mathrm{~dB}$ after three reversals. The equal loudness value for each replication was determined by an average of at least the last four reversals (or in some cases an even number of reversals greater than four). The reported equal loudness value was recorded as an average of estimates from three consecutive replications of the procedure. The outcome of this task was the HA loudness level which was perceived as equal to the CI MCL.

\section{Lateralization Task}

The purpose of this task was to estimate the IDD using listeners' capacity to detect changes in ITD. It was predicted that most listeners would have difficulty identifying changes in ITD because of a lack of frequencymatched inputs (Francart and Wouters, 2007; Francart 
et al, 2009a; 2011b; Goupell et al, 2013) or from distortions of the ITD by the HA and/or the CI (van Hoesel, 2012). It is also possible that the high and constant channel stimulation rates used by CI sound processors may have exceeded the rates usable for ITD resolution. On the other hand, the ability to consistently complete the task might indicate the existence of an overlapping region of frequency-matched neurons between the HA and CI ears.

Signals previously determined to be balanced in loudness at the listeners MCL were presented simultaneously and in a left/right adaptive procedure; listeners were asked to identify from which ear the sound seemed to originate. If the listener perceived that the sound source was on the HA side, the electric delay was decreased and if it was perceived that the sound source was on the CI side, the electric delay was increased. The step size was $0.3 \mathrm{msec}$ for the first two reversals and $0.05 \mathrm{msec}$ for the remainder of the procedure until an additional four reversals had been obtained; the last four reversals were averaged to obtain the value for each replication. The procedure was repeated three times; values obtained from the three replications were averaged to identify a final IDD. This value represents the delay between the HA and CI processors that the listener needed to achieve a percept of a centered single stimulus (i.e., eliminate lateralization due to device asynchrony). Performance for listeners who reported no change in lateralization of the sound with a maximum ITD of $2 \mathrm{msec}$, or who were unable to lateralize consistently (varying by $0.5 \mathrm{msec}$ or more across three trials), was characterized as without sensitivity to ITD.

The evidence basis for establishing a reasonable or expected IDD value to synchronize bimodal devices is not yet documented. Much of the literature investigating bimodal and bilateral sensitivity to timing delays includes direct, computer-controlled stimuli to specific locations along the electrode array. Nonetheless, it was predicted that after $2 \mathrm{msec}$, which is more than twice the largest typically occurring ITD produced by the human head of about $700 \mu \mathrm{s}$, the likelihood that listeners were hearing two auditory stimuli rather than one fused stimulus would increase and consequently introduce greater ambiguity in the use of location cues during the MAA task.

\section{MAA Task}

The purpose of this task was to determine whether listeners could perceive differences in spatial location and to measure the smallest perceivable angle separating two sources that listeners could discriminate after the HA and CI are loudness balanced without and with the IDD. Listeners completed a MAA task where both ITDs and ILDs were available, and without level roving, meaning that monaural loudness cues could also be used to complete the task. The purpose of this was to maximize above chance performance, which would potentially allow us to better show the effect of the IDD correction and provide a more ecologically valid testing scenario. The stimuli consisted of binaurally recorded speech sounds that were presented virtually using a method of constant stimuli. Listeners completed one session of practice trials in each condition to become acclimated to the task. In condition 1, the CI-HA loudness-balanced stimuli were used. In condition 2 , the same stimuli were used in addition to an IDD to compensate for the interaural processing delay.

Listeners were asked to listen to three presentations of "hawed" and to identify which presentation came from a location different from the other two. The randomized odd-ball presentation of "hawed" always came from the listeners' CI ear (located at an azimuth ranging from $0^{\circ}$ to $90^{\circ}$ ) and the other two reference presentations always came from a $0^{\circ}$ reference location. Therefore, listeners compared presentations of "hawed" separated by $90^{\circ}, 78.75^{\circ}$, and $67.5^{\circ}$. The listeners were presented 20 trials per spatial location. To avoid fatigue and diminished attention, the 20 trials were divided into two sets of ten. If results indicated above chance performance (i.e., percent correct $>54 \%$, based on $95 \%$ confidence interval for chance performance $=33 \%$ and 20 trials) in at least one of these spatial locations, the listener was asked to compare more difficult angles separated by $56.25^{\circ}, 45^{\circ}$, and $33.75^{\circ}$. If listeners continued to perform well on the task, the task was completed in the most difficult comparisons including $22.5^{\circ}$ and $11.25^{\circ}$ from the $0^{\circ}$ reference location.

Listeners who could not lateralize the signal only completed the MAA task in condition 1. Listeners who could consistently identify an IDD in the lateralization task completed the MAA task in conditions 1 and 2 in a random order. Breaks were provided between each condition.

\section{RESULTS}

\section{Loudness-Balancing Task}

Figure 2A depicts averaged loudness differences between the CI MCL and the acoustic input identified as equally loud for each listener. All listeners required increased acoustic input relative to the CI MCL to achieve equal loudness between ears. Large variation in acoustic gain was observed with three listeners (B2, B3, and B7) requiring $<10 \mathrm{~dB}$ and four listeners (B1, B4, B5, and $\mathrm{B} 6$ ) requiring $>10 \mathrm{~dB}$, where one listener (B1) required up to $40 \mathrm{~dB}$ of acoustic gain in the $\mathrm{HA}$ ear to achieve equal loudness relative to the $\mathrm{CI}$ ear. A few listeners commented that the CI "overwhelmed" or "dominated" what was heard when listening binaurally. A Pearson correlation of CI-HA loudness difference and acoustic ear PTA was significant $(r=0.89, p=0.006)$. 
A

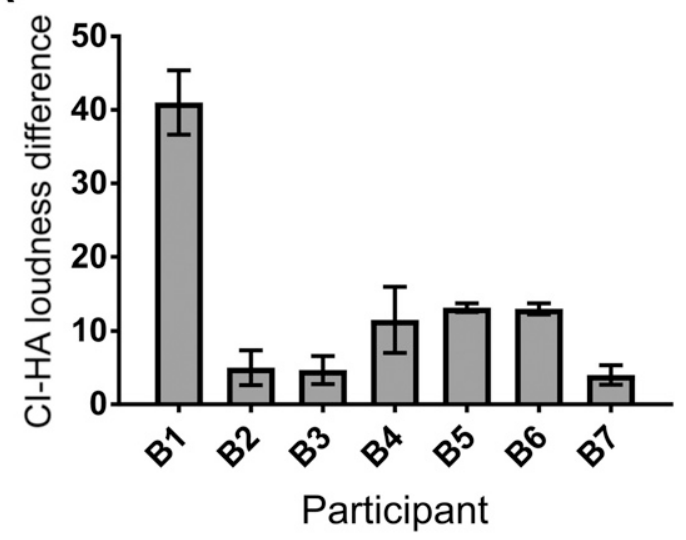

B

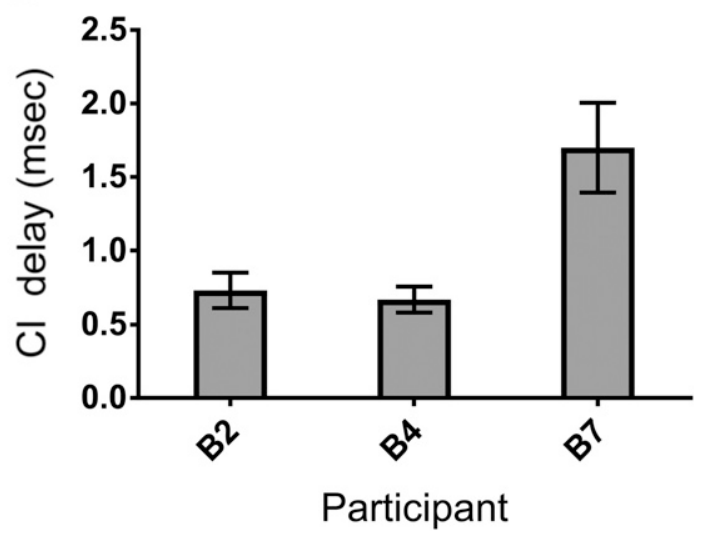

Figure 2. (A) Average differences between the MCL in the CI ear and the acoustic level identified as equally loud, both presented through direct audio inputs where the CI MCL was the reference (with exception of B6, see "Discussion"). Error bars show \pm 1 standard error. (B) Averaged CI timing delay resulting in perceptual change in intracranial position for participant B2, B4, and B7. Error bars show \pm 1 standard error.

\section{Lateralization Task}

Listeners B1, B3, B5, and B6 could not lateralize the sound using ITDs because, by report, the intracranial position of the sound did not change with the maximum ITD of $2 \mathrm{msec}$ and/or delay values required for lateralization varied by $\geq 0.50$ msec across three trials. For these listeners, it was reported the sound always came from the CI side. Two of these listeners, B1 and B5, had high-frequency thresholds precluding aided audibility of high-frequency stimuli. Figure 2B shows the averaged IDD for the remaining three listeners (B2, B4, and B7) who could consistently lateralize the sound. For these listeners, the average IDDs (the hearing aid delay relative to the CI) were $0.70,0.73$, and 1.70 msec, respectively. Of note is that the two listeners with the lowest loudness difference between ears (B2 and B7) were two of the three listeners that could demonstrate some sensitivity to ITD.

\section{MAA Task}

Figure 3 shows MAA performance for five listeners who could reliably complete the task. The two listeners (B5 and B6) not represented in Figure 3 both subjectively and objectively were unable to discriminate the center auditory spatial location $\left(0^{\circ}\right)$ from the $90^{\circ}, 79^{\circ}$, and $67^{\circ}$ locations. Three listeners (B2, B3, and B4) could complete the task above chance level for at least some of the spatial locations. Interestingly, for the listeners who completed all angular comparisons (B2, B3, and B4), percent correct did not decrease with decreasing
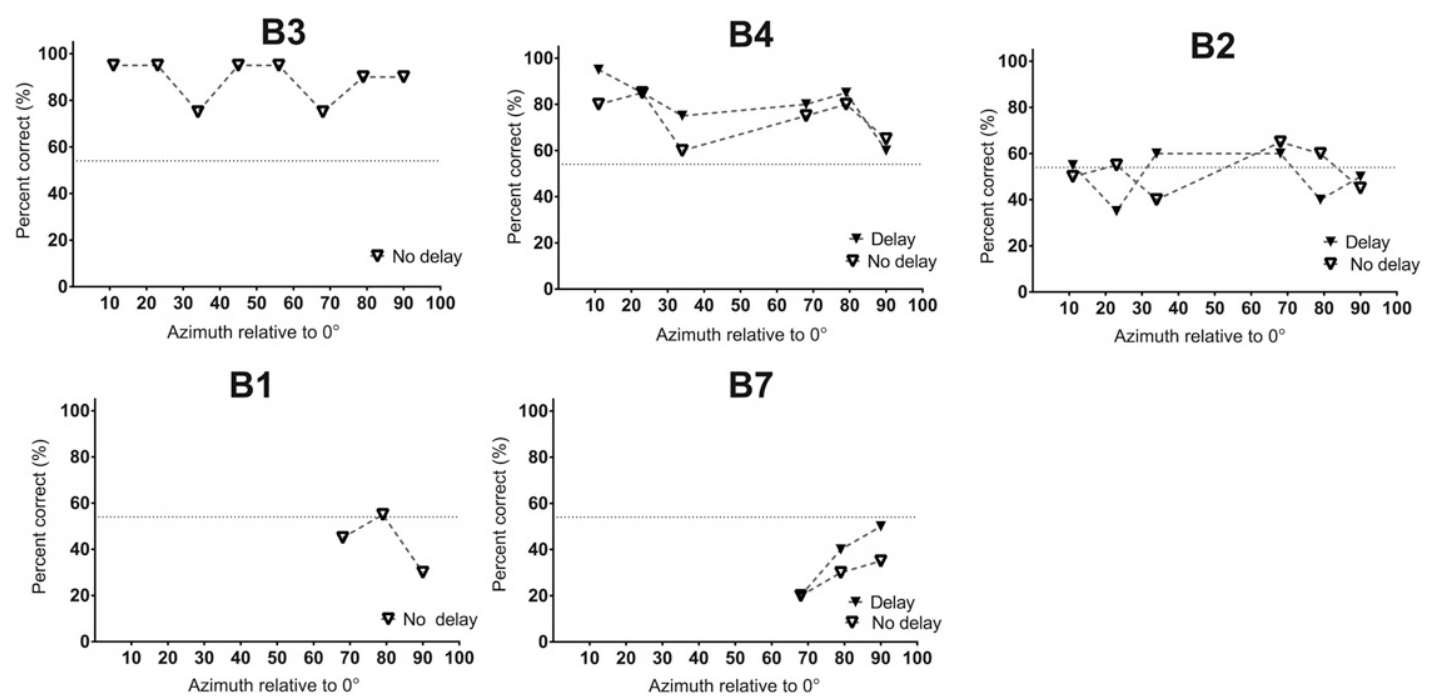

Figure 3. Individual mean MAA performance is shown in descending order based on accuracy with delay/no delay comparisons included for participants who could complete the lateralized task. Probability of above chance performance with $95 \%$ confidence intervals is denoted with the dotted line. 
angular separation; no significant differences were found between means for three easier $\left(90^{\circ}, 79^{\circ}\right.$, and $\left.67^{\circ}\right)$ and three more difficult $\left(33^{\circ}, 22^{\circ}\right.$, and $\left.11^{\circ}\right)$ conditions [paired two-tail $t$-test, $t_{(16)}=0.24, p=0.81$ ]. For further analysis, listeners were grouped based on sensitivity to ITD in condition 1.

No significant differences in mean peripheral angle $\left(90^{\circ}, 78^{\circ}\right.$, and $\left.67^{\circ}\right)$ MAA performance were found between listeners who were sensitive to ITD (B2, B4, and $\mathrm{B} 7$; mean $=55.6$, standard deviation $[\mathrm{SD}]=$ 25.2) and those determined to be insensitive to ITD [B1 and B3; mean $=64.2$, SD $=24.0$; paired, two-tail $t$-test; $\left.t_{(13)}=0.66, p=0.51\right]$. Similarly, for these five listeners, no significant relationship was found between the MAA performance and the CI-HA loudness difference $(r=-0.26, p=0.67)$.

In condition 2 (Figure 3 ), the three listeners who could lateralize the signal (B2, B4, and B7) repeated the MAA task with an IDD correction applied in an attempt to perceptually synchronize two devices. For each condition, average percent correct was calculated for each listener across spatial locations and the difference was tested with a paired two-tail $t$-test. No significant effect of IDD correction on spatial acuity was found when we compared group mean MAA error collapsed across peripheral angle comparisons [with IDD (mean $=54.9, \mathrm{SD}=1.0)$ and without IDD (mean $=$ $\left.52.8, \mathrm{SD}=4.2) ; t_{(2)}=0.55, p=0.63\right]$. It should be noted that for the listener with the most consistent and accurate MAA performance (B3), the IDD correction could not be established and the delay correction could not be applied.

\section{DISCUSSION}

$\mathrm{T}$ he purpose of this study was to determine if accounting for interdevice loudness differences and IDDs might result in improved perception of spatial differences in a MAA task. We measured MAA performance with HA and CI devices balanced in loudness both with and without an IDD correction. We hypothesized that the listeners able to lateralize the signals based on changes in IDD would be better able to discriminate auditory spatial location. We further hypothesized that the inclusion of the IDD correction would optimize acoustic and electric synchrony and improve MAA performance. Our results were not consistent with these hypotheses. Only three of seven listeners could perform the ITD lateralization task to measure an IDD. Only three listeners could perform the MAA task reliably above chance level. The latter result was surprising, given that the listeners seemingly had access to monaural level cues to perform the task. Furthermore, IDD correction provided no consistent improvement to MAA performance for any individual listener. These results suggest that conveying localization information to bimodal listeners necessitates more substantial changes to device speech processing algorithms and audiological fitting procedures than were conducted in this study.

\section{Interdevice Loudness}

Generally, all listeners reported difficulty and showed high variability in the loudness-balancing task. Figure 2A shows variations in average intensity-level differences between the CI ear MCL and HA ear to achieve equal loudness. It is possible that listeners with lower acoustic thresholds and smaller loudness differences between ears were either the recipients of better fit/mapped devices and/or had hearing configurations that afforded more overlap to better accommodate combined acoustic and electric stimulation. These ideas are consistent with recent data showing advantages of HA-aided thresholds (better than $45 \mathrm{~dB} \mathrm{HL}$ ) for bimodal listener sound source direction sensitivity when automatic gain compression controls are matched between ears (Veugen et al, 2016a).

\section{Interdevice Delay Measurements}

Only three listeners could use the IDD to consistently lateralize the stimuli (Figure 2B). The reported difficulty and the high intra- and interlistener variability during these tasks provide evidence that compensating for CI and HA device delays may not be useful for optimizing interaural comparisons of broadband stimuli.

\section{Group Effects on Detection of Spatial Differences}

Our data suggest that most listeners could not differentiate sound source locations consistently, in agreement with other reports including bimodal listeners (Potts and Litovsky, 2014; Dorman et al, 2016). During the MAA task without the IDD, only three of the seven listeners could consistently perform above chance level, which is surprising based on the availability of monaurallevel cues. For the few listeners who could complete the MAA task with consistency, their strategy for doing so is not clear. One possibility is that listeners could extract monaural loudness cue information. Neither the addition of the IDD correction nor changes in reference angle significantly influenced MAA performance, which is suggestive of a nonbinaural strategy.

The use of monaural cues to perform tasks that are thought to be binaural in nature has been studied in normal-hearing listeners and in listeners with unilateral impairments (Shub et al, 2008). In that study, both groups could reliably complete monaural MAA localization tasks with RMS errors of $\sim 30^{\circ}$ when feedback was provided, and there were no consistent differences in 
localization performance noted between the normal-hearing listeners who listened with only one ear and the listeners with unilateral hearing loss.

In the present study, it was thought that listeners with the smallest differences in interaural loudness perception might be best able to use monaural loudness cues in the MAA task. Listeners B2 and B3 support this idea, but B7 does not (Figure 3). While identifying the louder ear might have been a viable strategy theoretically, it is possible that the means by which HA and CI devices transmit the high-frequency information supporting ILD cues was too dissimilar, and differences in automatic gain controls diminished these potential loudness cues. Synched automatic gain controls (Veugen et al, 2016a) or enhancement of ILDs at low frequencies (Brown, 2013) may be viable processing strategy approaches to this problem.

Differences in hearing history have been used to explain variation in CI listener binaural listening ability. For example, sensitivity to ITD has been shown in bilateral CI listeners who were postlingually but usually not prelingually deafened (Litovsky et al, 2010; see also; Goupell, 2015). In the present study, listeners B4 and B5, who have congenital deafness and high acousticear pure-tone averages $(\sim 88$ and $76 \mathrm{~dB} \mathrm{HL})$, provide an interesting contrast. Despite the similarity in hearing history in these two listeners, B4 showed superior performance in the MAA and lateralization tasks. It is possible that the early age of intervention might have played a role in preserving some of B4's sensitivity to binaural cues in comparison to B5 who was identified at a later age.

CIs stimulate neural regions corresponding to higher frequencies most effectively, which is where the input of HAs is sometimes limited. The interaurally overlapping frequency ranges necessary to compute binaural cues may also be limited by hearing loss configuration. The three listeners (B2, B3, and B4) who met the $65 \%$ criteria in the MAA task and moved to more difficult angular comparisons all have relatively flat hearing losses generally within the moderate to severe hearing loss range. It is possible that these three listeners accessed a greater range of useable acoustic input overlapping with electrically stimulated information to provide more meaningful interaural intensity or temporal comparisons. This finding is supported by Francart (2009b) who found that for bimodal listeners, if the average threshold of the residual hearing at 1 and $2 \mathrm{kHz}$ is better than about $100 \mathrm{~dB} \mathrm{HL}$, lateralization is possible with ITD cues. Although the findings of Francart (2009b) were obtained with direct electrical stimulation (i.e., bypassing the CI sound processor and controlling the CI with a computer), the contribution of the HA in the present study may have allowed access to ILDs or envelope ITDs. It should be mentioned that one listener (B6), with a similar flat, moderately severe to profound hearing loss could not lateralize or complete the MAA task. This listener demonstrated clear preference for the HA in comparison to the CI which may have contributed to a limited ability to access interaural cues for comparison.

It may also be that the potential overlapping frequency region is at nonoptimal frequencies. First, ITDs are mostly limited to $<1400-1500 \mathrm{~Hz}$ (Brughera et al, 2013). Second, ILDs are nonmonotonic functions of azimuth between 1000 and $4000 \mathrm{~Hz}$ (Macaulay et al, 2010) because of an acoustical bright spot and constructive interference, meaning that ILDs become smaller for increasing azimuth beyond $\sim 45^{\circ}$. It may be that even if bimodal listeners had hearing in this frequency range, it may provide little useful, or even conflicting, location information. Previous studies have shown difficulties in localizing sounds (Stevens and Newman, 1936) and performing MAA discriminations (Mills, 1958) in this frequency range.

\section{Individual Performance Data}

Listener B1 reported two years of CI listening experience and comparatively showed the most severe degree of hearing loss in the acoustic ear. This listener anecdotally described most benefit in the bimodal condition for awareness of environmental sound and reported that the CI often dominated the HA. This considerable difference in loudness percept between ears was demonstrated during the loudness-balancing task (Figure 2A). This listener required up to $40 \mathrm{~dB}$ of acoustic gain to achieve equal loudness relative to the $\mathrm{CI}$ ear. B1 was unable to perceive an IDD consistently and demonstrated at or below chance performance in the easiest MAA task comparisons (Figure 3A).

Listener B2 reported five years of consistent CI use in addition to a HA for improved speech understanding, environmental awareness, and to better appreciate music. This listener's moderately severe sloping to severe acoustic threshold configuration was relatively flat in comparison to the other listeners tested. In both the loudness-balancing and delay tasks, B2 could demonstrate relative consistency; the loudness difference values between ears were among the lowest of the group (Figure 2A). During the MAA task, this listener showed a pattern of relatively high accuracy in the peripheral angular comparisons $\left(79^{\circ}, 67^{\circ}, 56^{\circ}\right.$, and $45^{\circ}$ ), with exception of the $90^{\circ}$ comparison, and showed difficulty in the central angular comparisons (Figure 3). When the MAA task was repeated with an IDD correction, there was limited evidence to suggest that B2 benefitted from the attempt to better synchronize devices $\left(11.25^{\circ}\right.$ and $\left.33.75^{\circ}\right)$.

A long-term HA user with acoustic thresholds in the severe to profound hearing loss range, B3, reported consistent use of the CI in combination with the HA for the past three years. Although B3 reported difficulty comparing loudness levels between ears, the loudness 
differences between devices were among the smallest. The addition of an electric delay had no effect on the intracranial position of the stimuli for this listener. During the MAA task, B3 showed consistently high accuracy in most angular comparisons (Figure 3 ). When completing the MAA task weeks later, this consistently high performance across angular comparisons was confirmed. Interestingly, B3's high performance remained accurate, even in the more difficult, central angular comparisons. This finding is surprising if we assume that the use of interaural cues would make these comparisons easier at larger angles and more difficult at smaller angles. The fact that B3's performance remained consistently high might suggest this listener was relying on monaural cues that are less likely to diminish with decreasing angular separation in the more central angular comparisons or that smaller separations were necessary to show a decrease in performance.

As the youngest of listeners included in this study, B4 reported approximately five years of CI listening experience and with long-term HA use. This listener's congenital hearing loss was identified at a very young age, at which time B4 began using HAs. Acoustic hearing thresholds for this listener fell within the moderate to severe hearing loss range. Although describing overall bimodal benefit (i.e., the HA provides "fullness and depth to speech"), this listener anecdotally reported sound source localization limitations beyond coarse left/right hemifield representation. This listener perceived the $\mathrm{CI}$ as sounding louder and showed higher variability in the loudnessbalancing task relative to other listeners, which may suggest this was a more difficult task. The lateralization task was completed with relative consistency, and MAA performance remained relatively high across all angular comparisons. Interestingly, MAA accuracy did not decrease as one might expect in the more difficult peripheral angular comparisons. In most of the MAA angular comparisons tested with the IDD correction, this listener showed slight improvement. Although this listener reported a congenital hearing loss, it is possible that the early age of HA intervention in combination with consistent CI listening training better preserved neural integrity, which could have affected MAA performance.

Listener B5 also had a congenital hearing loss, identified around eight years of age, at which time HA use started. This listener reportedly listened bimodally for the past 16 years, but described the CI as sounding "mechanical." Contralateral ear audiometric testing shows moderate low-frequency sloping to profound sensorineural hearing loss. Both the loudness-balancing and the lateralization tasks were difficult for this listener based on the perception that the sound was always louder and originated from the CI side. The island of low-frequency hearing might suggest that this listener had the best opportunity to extract ITDs that might help distinguish spatial locations. However, in the
MAA task, this listener was unable to distinguish angular locations with $90^{\circ}$ separation. It is possible that childhood years of auditory deprivation limited the functional utility of existing low-frequency acoustic hearing.

Listener B6 had the least amount of experience listening bimodally, with around ten years of HA use and approximately one year of CI use. This listener reported heavy reliance on the HA in all listening situations with moderately severe to severe contralateral acoustic hearing sensitivity. Initially, during the loudness-balancing task, this listener reported difficulty comparing loudness levels between ears and was highly variable in identifying an MCL in the CI ear. Based on the difficulty establishing an MCL in the CI ear, the MCL in the HA ear was identified and used as a reference in the loudness-balancing task. This listener reported that the loudness-balancing task was difficult because sounds were too different to compare. Lateralization tasks were attempted with the addition of a delay in the CI ear and in the HA ear with reports of no change in intracranial position. Like B5, this listener's MAA performance showed very limited ability to distinguish angular locations in the easiest comparisons $\left(0^{\circ}\right.$ in comparison to $\left.90^{\circ}\right)$. It is possible that this listener's limited CI listening experience contributed to the difficulty demonstrated in all listening tasks.

Listener B7 also reported relatively limited bimodal listening experience of two years, with longer term HA experience of 22 years. This listener reportedly listened consistently in the bimodal condition, with mild sloping to profound contralateral acoustic thresholds. In comparison to the other listeners, B7 perceived the smallest intensity difference between ears at the MCL. This listener could perceive a change in intracranial position with an electric delay, although performance was more variable in comparison to B2 and B4 (Figures $2 \mathrm{~A}$ and B). Despite the sensitivity demonstrated in these tasks, this listener showed difficulty completing the MAA tasks in the easiest conditions with performance around chance level.

There are a few limitations to the present study. All listeners were fit with a Phonak Naida UP IX HA to represent a bimodal configuration currently used by patients, while also controlling for major differences in listener's own HA technology. The CI MCL was identified before adjusting the contralateral HA to a level equal in loudness. Because the actual range of levels used, as computed via direct audio input to sound pressure level, was not measured, it is difficult to estimate the amount of compression incurred for each device in each listener. Nonetheless, it is estimated that listeners likely identified MCL at a level similar to normal, conversational speech, which is expected to be below the level that produces infinite compression. 
Variability in degree of hearing loss and in reported MCL meant that each listener completed the lateralization and MAA tasks at different presentation levels, which likely means that the level of compression was different for each listener. Differences in compression may have contributed to greater variability in loudness perception. Because compression ratios vary as a function of hearing loss, this problem may have been minimized to some degree had we focused on bimodal listeners with defined degree of residual acoustic hearing loss. Another attempt at resolving this issue may have been to alter the fitting formula; however, to explore which temporal or intensity characteristics are perceptible through currently available clinical processors programmed as recommended by the manufacturer, we decided not to alter the programming.

Another limitation to the present study is that amplification was not adjusted to match the targets of validated prescriptive methods using real-ear verification data. We initially validated that the fitting of the HA resulted in a comfortable level through subjective report and further attempted to match loudness through psychophysical means (i.e., left/right adaptive loudness comparison). Inclusion of real-ear verification would have undoubtedly strengthened the fitting procedures of this study. In an effort to improve this aspect, the authors retroactively obtained simulated real-ear measures using average adult real-ear to coupler difference values (Ching and Dillon, 2003) and each individual participant's acoustic audiometric thresholds measured at the time of testing. Output levels were measured on a Fonix 7000 hearing aid analyzer (Frye Electronics, Inc.) the Frye Box using a 60-dB SPL composite input signal and compared with Phonak propriety standard fitting targets. Simulated gain measures verified audibility above targets at $0.25,0.5$, and $1 \mathrm{kHz}$ for all participants with exception of B1 (with limited audibility at $1 \mathrm{kHz}$ ). For three participants (B4, B4, and B6), acoustic audibility was extended from 0.25 to $3 \mathrm{kHz}$. For one participant (B2), acoustic audibility was extended from 0.25 to $4 \mathrm{kHz}$. For all participants, the simulated acoustic gain approximated at the frequencies of the speech stimuli $\left(F_{0}\right.$ of $205 \mathrm{~Hz}$, and formant frequencies of 408,1042, 1600, and $2803 \mathrm{~Hz}$ ) was greater than that required for loudness balancing with the CI ear. It should also be noted that in the CI ear, we did not measure aided thresholds before the experiment. Because of this failure to document CI-aided thresholds, neither audibility (overall or across-frequency) nor best fitting clinical practices can be verified. We assumed that programming parameters in this ear were appropriate for audibility based on speech-perception performance.

Other limitations of this study include the relatively small sample size, and the reduced subset of participants with measurable and usable higher frequency acoustic hearing. The proposed hypotheses would have best been tested had there been greater overlap in the frequencies of usable hearing and that of the broadband speech stimulus. Under ideal circumstances, data collection would have taken place for listeners with similar bimodal listening experience.

\section{CONCLUSION}

$\Upsilon$ his study shows that for most of the bimodal lis1 teners tested, it was both subjectively (as anecdotally reported) and objectively (per MAA task score) difficult to differentiate a signal presented from $0^{\circ}$ and $90^{\circ}$, even after attempting to compensate for IDDs and loudness differences. A few listeners could distinguish differences in sound source location across all azimuth positions, as small as between $0^{\circ}$ and $11.25^{\circ}$.

The listeners who could complete the MAA task with accuracy may have done so via binaural processing of ITDs and/or ILDs. For listeners with usable low-tomid frequency acoustic hearing, CI and HA stimulation patterns might allow for benefit from tonotopic overlap. This overlap in stimulation may facilitate perceptual fusion between ears and the ability to distinguish the sounds at different spatial locations. Alternatively, listeners who consistently completed the MAA task may have extracted monaural loudness cues. A monaural strategy was the more likely explanation based on the high variability and general difficulty demonstrated in tasks requiring binaural comparisons. Between-ear differences in automatic gain compression likely limited the ILD cue that might have been useful for differentiating angular location and may have rendered monaural extraction of loudness cues a more useful strategy.

We conclude that bimodal listeners may benefit from bimodal processing, but fitting strategies need to convey spatial cues with better fidelity. Future research should elaborate on these findings with greater attention to providing the opportunity for useful binaural comparisons. More useful binaural comparisons should support superior functional listening advantages more so than the monaural listening strategies likely used in this study.

\section{REFERENCES}

Armstrong M, Pegg P, James C, Blamey P. (1997) Speech perception in noise with implant and hearing aid. Am J Otol 18(6, Suppl):S140-S141.

Aronoff JM, Freed DJ, Fisher LM, Pal I, Soli SD. (2011) The effect of different cochlear implant microphones on acoustic hearing individuals' binaural benefits for speech perception in noise. Ear Hear 32(4):468.

Bernstein JG, Goupell MJ, Schuchman GI, Rivera AL, Brungart DS. (2016) Having two ears facilitates the perceptual separation of concurrent talkers for bilateral and single-sided deaf cochlear implantees. Ear Hear 37(3):289.

Brown CA. (2013) Binaural enhancement for bilateral cochlear implant users. Ear Hear 35(5):580-584. 
Brughera A, Dunai L, Hartmann WM. (2013) Human interaural time difference thresholds for sine tones: the high-frequency limit. $J$ Acoust Soc Am 133(5):2839-2855.

Ching TY, Dillon H. (2003) Prescribing amplification for children: adult-equivalent hearing loss, real-ear aided gain, and NAL-NL1. Trends Amplif 7(1):1-9.

Ching TYC, Incerti P, Hill M. (2004) Binaural benefits for adults who use hearing aids and cochlear implants in opposite ears. Ear Hear 25:9-21.

Ching TYC, Van Wanrooy E, Dillon H. (2007) Binaural-bimodal fitting or bilateral implantation for managing severe to profound deafness: a review. Trends Amplif 11(3):161-192.

Crew JD, Galvin JJ, III, Landsberger DM, Fu QJ. (2015) Contributions of electric and acoustic hearing to bimodal speech and music perception. PLoS One 10(3):e0120279.

Devocht EM, Janssen AML, Chalupper J, Stokroos RJ, George EL. (2017) The benefits of bimodal aiding on extended dimensions of speech perception: intelligibility, listening effort, and sound quality. Trends Hear 21:2331216517727900.

Dorman MF, Gifford RH. (2010) Combining acoustic and electric stimulation in the service of speech recognition. Int $J$ Audiol 49(12): 912-919.

Dorman MF, Loiselle LH, Cook SJ, Yost WA, Gifford RH. (2016) Sound source localization by normal-hearing listeners, hearing-impaired listeners and cochlear implant listeners. Audiol Neurotol 21(3):127-131.

Dunn C, Tyler R, Witt S. (2005) Benefit of wearing a hearing aid on the unimplanted ear in adult users of a cochlear implant. J Speech Lang Hear Res 48:668-680.

Feddersen WE, Sandel TT, Teas DC, Jeffress LA. (1957) Localization of high-frequency tones. J Acoust Soc Am 29(9):988-991.

Francart T, Van den Bogaert T, Moonen M, Wouters J. (2009a) Amplification of interaural level differences improves sound localization in acoustic simulations of bimodal hearing. J Acoust Soc Am 126(6):3209-3213.

Francart T, Brokx J, Wouters J. (2009b) Sensitivity to interaural time differences with combined cochlear implant and acoustic stimulation. J Assoc Res Otolaryngol 10(1):131-141.

Francart T, Lenssen A, Wouters J. (2011a) Enhancement of interaural level differences improves sound localization in bimodal hearing. J Acoust Soc Am 130(5):2817-2826.

Francart T, Lenssen A, Wouters J. (2011b) Sensitivity of bimodal listeners to interaural time differences with modulated single-and multiple-channel stimuli. Audiol Neurotol 16(2):82-92.

Francart T, Lenssen A, Wouters J. (2014) Modulation enhancement in the electrical signal improves perception of interaural time differences with bimodal stimulation. J Assoc Res Otolaryngol 15(4):633-647.

Francart T, McDermott HJ. (2013) Psychophysics, fitting, and signal processing for combined hearing aid and cochlear implant stimulation. Ear Hear 34(6):685-700.

Francart T, Wouters J. (2007) Perception of across-frequency interaural level differences. J Acoust Soc Am 122(5):2826-2831.

Fu QJ, Chinchilla S, Galvin JJ. (2004) The role of spectral and temporal cues in voice gender discrimination by normal-hearing listeners and cochlear implant users. $J$ Assoc Res Otolaryngol $5(3): 253-260$.
Fu QJ, Nogaki G, Galvin JJ. (2005) Auditory training with spectrally shifted speech: implications for cochlear implant patient auditory rehabilitation. J Assoc Res Otolaryngol 6(2):180-189.

Gfeller K, Turner C, Oleson J, Zhang X, Gantz B, Froman R, Olszewski C. (2007) Accuracy of cochlear implant recipients on pitch perception, melody recognition, and speech reception in noise. Ear Hear 28:412-423.

Goupell MJ. (2015) Interaural envelope correlation change discrimination in bilateral cochlear implantees: effects of mismatch, centering, and onset of deafness. J Acoust Soc Am 137(3): $1282-1297$.

Goupell MJ, Stoelb C, Kan A, Litovsky RY. (2013) Effect of mismatched place-of- stimulation on the salience of binaural cues in conditions that simulate bilateral cochlear-implant listening. $J$ Acoust Soc Am 133(4):2272-2287.

Hamzavi J, Marcel Pok S, Gstoettner W, Baumgartner WD. (2004) Speech perception with a cochlear implant used in conjunction with a hearing aid in the opposite ear. Int $J$ Audiol 43(2):61-65.

Hillenbrand J, Getty LA, Clark MJ, Wheeler K. (1995) Acoustic characteristics of American English vowels. J Acoust Soc Am 97(5): 3099-3111.

Kayser H, Ewert SD, Anemuller J, Rohdenburg T, Hohmann V, Kollmeier B. (2009) Database of multichannel in-ear and behind-the-ear head-related and binaural room impulse responses. EURASIP J Adv Signal Process 2009:1-10.

Kokkinakis K, Pak N. (2014) Binaural advantages in users of bimodal and bilateral cochlear implant devices. J Acoust Soc Am 135(1):EL47-EL53.

Kwon BJ. (2012) AUX: a scripting language for auditory signal processing and software packages for psychoacoustic experiments and education. Behav Res Methods 44(2):361-373.

Landsberger DM, Svrakic Svrakic J, Svirsky M. (2015) The relationship between insertion angles, default frequency allocations, and spiral ganglion place pitch in cochlear implants. Ear Hear 36(5):e207-e213.

Lenssen A, Francart T, Brokx J, Wouters J. (2011) Bimodal listeners are not sensitive to interaural time differences in unmodulated low-frequency stimuli (L). J Acoust Soc Am 129(6):34573460

Litovsky RY, Jones GL, Agrawal S, van Hoesel R. (2010) Effect of age at onset of deafness on binaural sensitivity in electric hearing in humans. J Acoust Soc Am 127:400-414.

Macaulay EJ, Hartmann WM, Rakerd B. (2010) The acoustical bright spot and mislocalization of tones by human listeners. $J$ Acoust Soc Am 127(3):1440-1449.

Macpherson EA, Middlebrooks JC. (2002) Listener weighting of cues for lateral angle: the duplex theory of sound localization revisited. J Acoust Soc Am 111(5):2219-2236.

Majdak P, Goupell MJ, Laback B. (2011) Two-dimensional localization of virtual sound sources in cochlear-implant listeners. Ear Hear 32(2):198-208.

Mills AW. (1958) On the minimum audible angle. J Acoust Soc Am 30(4):237-246.

Mills AW. (1959) Thresholds for interaural difference in intensity. $J$ Acoust Soc Am 31(6):830. 
Morera C, Manrique M, Ramos A, Garcia-Ibanez L, Cavalle L, Huarte A, Estrada E. (2005) Advantages of binaural hearing provided through bimodal stimulation via a cochlear implant and a conventional hearing aid: a 6-month comparative study. Acta Otolaryngol 125(6):596-606.

Most T, Gaon-Sivan G, Shpak T, Luntz M. (2011) Contribution of a contralateral hearing aid to perception of consonant voicing, intonation, and emotional state in adult cochlear implantees. J Deaf Stud Deaf Educ 17(2):244-258.

Nittrouer S, Chapman C. (2009) The effects of bilateral electric and bimodal electric - acoustic stimulation on language development. Trends Amplif 13(3):190-205.

Olson AD, Shinn JB. (2008) A systematic review to determine the effectiveness of using amplification in conjunction with cochlear implantation. J Am Acad Audiol 19(9):657-671.

Potts LG, Litovsky RY. (2014) Transitioning from bimodal to bilateral cochlear implant listening: speech recognition and localization in four individuals. J Am Acad Audiol 23(1):79-92.

Potts LG, Skinner MW, Litovsky RA, Strube MJ, Kuk F. (2009) Recognition and localization of speech by adult cochlear implant recipients wearing a digital hearing aid in the nonimplanted ear (bimodal hearing). J Am Acad Audiol 20(6):353-373.

Sampaio AL, Araújo MF, Oliveira CA. (2011) New criteria of indication and selection of patients to cochlear implant. Int J Otolaryngol 2011:I573968.

Schafer EC, Amlani AM, Paiva D, Nozari L, Verret S. (2011) A meta-analysis to compare speech recognition in noise with bilateral cochlear implants and bimodal stimulation. Int $J$ Audiol 50(12): 871-880.

Seeber B, Baumann U, Fastl H. (2004) Localization ability with bimodal hearing aids and bilateral cochlear implants. J Acoust Soc Am 116:1698-1709.

Shallop JK, Arndt PL, Turnacliff KA. (1992) Expanded indications for cochlear implantation: perceptual results in seven adults with residual hearing. J Speech-Language Pathol Audiol 16(2):141-148.

Shaw EAG, Vaillancourt MM. (1985) Transformation of soundpressure level from the free field to the eardrum presented in numerical form. J Acoust Soc Am 78:1120-1123.
Shub DE, Carr SP, Kong Y, Colburn HS. (2008) Discrimination and identification of azimuth using spectral shape. J Acoust Soc Am 124(5):3132-3141.

Stevens SS, Newman EB. (1936) The localization of actual sources of sound. Am J Psychol 48(2):297-306.

Stone MA, Moore BC. (1999) Tolerable hearing aid delays. I. Estimation of limits imposed by the auditory path alone using simulated hearing losses. Ear Hear 20(3):352-388.

Stone MA, Moore BC. (2003) Tolerable hearing aid delays. III. Effects on speech production and perception of across-frequency variation in delay. Ear Hear 24(2):175-183.

Sucher CM, McDermott HJ. (2009) Bimodal stimulation: benefits for music perception and sound quality. Cochlear Implants Int 10(S1):96-99.

Svirsky MA, Silveira A, Neuburger H, Teoh SW, Suarez H. (2004) Long-term auditory adaptation to a modified peripheral frequency map. Acta Otolaryngol 124(4):381-386.

van Hoesel RJ. (2012) Contrasting benefits from contralateral implants and hearing aids in cochlear implant users. Hear Res 288(1):100-113.

Veugen LC, Chalupper J, Snik AF, van Opstal AJ, Mens LH. (2016a) Matching automatic gain control across devices in bimodal cochlear implant users. Ear Hear 37(3):260-270.

Veugen LC, Hendrikse MM, van Wanrooij MM, Agterberg MJ, Chalupper J, Mens LH, van Opstal AJ. (2016b) Horizontal sound localization in cochlear implant users with a contralateral hearing aid. Hear Res 336:72-82.

Von Ilberg CA, Baumann U, Kiefer J, Tillein J, Adunka OF. (2011) Electric-acoustic stimulation of the auditory system: a review of the first decade. Audiol Neurotol 16(2, Suppl):1-30.

Wightman FL, Kistler DJ. (1992) The dominant role of lowfrequency interaural time differences in sound localization. $J$ Acoust Soc Am 91(3):1648-1661.

Zhang T, Spahr AJ, Dorman MF, Saoji A. (2013) The relationship between auditory function of non-implanted ears and bimodal benefit. Ear Hear 34:133-141. 